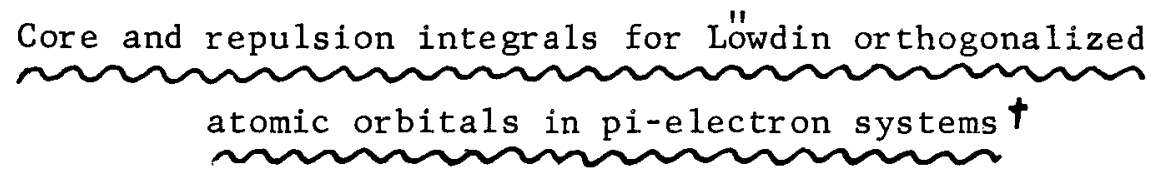

by

D. P. CHONG

University of Wisconsin Theoretica1 Chemistry Institute Madison, Wisconsin

$$
30463
$$

The core integrals and electron repulsion integrals for the $2 \mathrm{p} \pi$ Slater-type orbitals of the ally1 radical, trimethylenemethy1 radica1, and benzene molecule have been transformed to the corresponding Löwdin or thogonalized basis set of atomic orbitals. A11 the transformed multi-center non-Coulomb repulsion integrals are found to have reduced in magnitude.

† This research was supported by the following grant: Nationa1 Aeronautics and Space Administration Grant NsG-275-62. 


\section{Introduction}

In quantum chemistry, orthogonalization of basis functions, when desired, is usually carried out by one of three we11-known methods. The most popular is the Schmidt process of orthogonalization [1]. If the orthogonalized orbitals $\psi_{l}$ is written as

$$
\psi_{\ell}=\sum_{k} \phi_{k} T_{k \ell},
$$

where $\underset{T}{\sim}$ is the transformation matrix and $\phi_{\mathrm{k}}$ are the original orbital functions, then the Schmidt transformation has zero $\mathrm{T}_{\mathrm{k} \ell}$ for $k>\ell$. In the second method of orthogonalization, one diagonalizes the overlap matrix $\underset{\sim}{\stackrel{S}{\sim}}$ by solving the eigenvalue problem

$$
[\stackrel{S}{\sim}-\lambda \underset{\sim}{\sim}] \underset{\sim}{\sim}=0
$$

If $\underset{\sim}{\sim}$ is the eigenfunction matrix, then $\underset{\sim}{\sim}$ is given by

$$
\mathrm{T}_{\mathrm{k} \ell}=\mathrm{F}_{\mathrm{k} \ell} \lambda_{\ell}^{-\frac{1}{2}}
$$

The third method, developed by Lowdin [2], starts with normalized orbitals, and $\mathrm{S}^{-\frac{1}{2}}$ is expanded in a power series in $(\underset{\sim}{\mathbf{N}}-\underset{\sim}{\sim})$. The resulting transformation matrix $\underset{\sim}{\sim}$ is symmetric. Carlson and Reller [3] have shown that the Lowdin orthogonalized orbitals resemble the original non-orthogonal orbitals most by a least 
squares criterion, that is

$\sum_{\mathrm{k}} \int\left|\psi_{\mathrm{k}}-\phi_{\mathrm{k}}\right|^{2} \mathrm{dv}=$ minimum

On the other hand, in theoretical investigations of $\pi$-electron systems, the approximation known as zero differential overlap (ZDO) has often been invoked, for example, in the simple Huckel molecular orbital method, or in the Pariser-Parr-Pople semi-empirical approach $[4,5]$.

The connection between the Lowdin orthogonalized atomic orbitals (LOAO) and the ZDO approximation has often been discussed, e.g. by Parr [6], and more recently by Lykos [7] and by Fischer-Hjalmas [8]. In the present investigation, the core integrals calculated by the method of Parr and Mulliken [9] and the electron repulsion integrals for the $\pi$-electron systems of the allyl radical $\mathrm{CH}_{2} \mathrm{CHCH}_{2}$, the trimethylenemethyl radical $\mathrm{C}_{\left(\mathrm{CH}_{2}\right)_{3}}$, and benzene $\mathrm{C}_{6} \mathrm{H}_{6}$ have been transformed to the corresponding LOAO basis. The radicals have been chosen because they represent two of the simplest $\pi$-electron systems with non-ionic resonance structures, and the electronic structure of their ground states have been fully investigated by Linnett and coworkers $[10-13]$. 


\section{Calculations}

The Hamiltonian operator in theoretical treatments of $\pi$-electron systems is usually written as [14]

$$
\mathcal{H}=\sum_{i} f(i)+\sum_{i<j} g(i, j),
$$

where

$$
\begin{aligned}
& f=t+\sum_{\nu} h_{\nu}, \\
& t=\text { kinetic energy operator, } \\
& h_{v}=\begin{array}{l}
\text { potential energy of electron in the } \\
\text { sigma framework due to nucleus } \nu,
\end{array}
\end{aligned}
$$

and, $g(i, j)=1 / r_{i j}$

If $\phi_{n}$ is the ordinary $2 p \pi$ atomic orbital on nucleus $n$, the following relation holds [9]:

$$
\left(t+h_{n}\right) \phi_{n}=w_{2 p} \phi_{n}
$$

where $W_{2 p}$ is the ionization potential of a $2 p \pi$ electron in a carbon atom in $\mathrm{sp}^{2}$ valence state. Let us define the following matrix elements, 


$$
\begin{aligned}
& s_{\mathrm{mn}}=\left\langle\phi_{\mathrm{m}}, \phi_{\mathrm{n}}\right\rangle, \\
& i_{\mathrm{mn}}=\left\langle\phi_{\mathrm{m}},\left(\sum_{\nu \neq \mathrm{n}} \mathrm{h}_{\nu}\right) \phi_{\mathrm{n}}\right\rangle,
\end{aligned}
$$

and,

$$
\mathrm{f}_{\mathrm{mn}}=\left\langle\phi_{\mathrm{m}}, f, \phi_{\mathrm{n}}\right\rangle
$$

Combining eqs. (2.2) to (2.7), one can easily obtain

$$
\stackrel{f}{\sim}=\mathrm{w}_{2 \mathrm{p}} \stackrel{s}{\sim}+\underset{i}{\sim}
$$

The matrix elements $f_{\mathrm{mn}}$ are called the core integrals [9], and have the values of $i_{m n}$, relative to $w_{2 p}$ as zero of energy. To avoid confusion, we shall call $f_{\mathrm{mn}}$ the total core integrals, and $i_{m n}$, the relative core integrals.

\subsection{Relative core integrals}

Using the Goeppert-Mayer and Sklar approximation [15], neglecting the hydrogen atoms, the relative core integrals can be evaluated [9] by

$$
i_{m n}=-\sum_{r \neq n}[(r: m n)+(m n ; r r)],
$$


where ( $r: m n)$ are the penetration integrals [15], and, the electron repulsion integrais are defined by

$$
(a b ; c d)=\left\langle\phi_{a}(1) \phi_{c}(2), g(1,2) \phi_{b}(1) \phi_{d}(2)\right\rangle
$$

There are available several tables of penetration integrals [16-21], which are either not sufficiently accurate or inconvenient for our use here. Therefore, they are recalculated with Sklar's formula [16], as corrected by Parr and Crawford [17]. The threecenter penetration integrals are approximated by

$$
(r: k \ell)=s_{k \ell}(r: m m),
$$

where $\phi_{\mathrm{m}}$ is a slater $2 \mathrm{p} \pi$ atomic orbital located midway between atoms $k$ and $\boldsymbol{l}$.

Throughout this work, slater $2 \mathrm{p} \pi$ atomic orbitals with $\mathrm{Z}=3.18$ are used, and a planar aromatic structure is assumed. The distance between neighboring nuclei is taken to be

$$
\frac{8.37}{3.18}=2.632075 \text { bohr, }
$$

a choice dictated by the availability [22] of the multi-center repulsion integrals.

The overlap integrals $s_{m n}$, calculated using Roothaan's formula [23], together with all the two-center penetration integrals needed, are listed in Table 1. 
All the repulsion integrals except one have been tabulated in the 1iterature $[13,22]$. The exception is $(11 ; 44)$ for benzene, which is evaluated with Roothaan's formula [23] to be 0.182605 hartree.

The relative core integrals $i_{m n}$, computed with eqs. (2.9) and (2.11), are listed in Table 2. The numbering in the three systems is as follows:
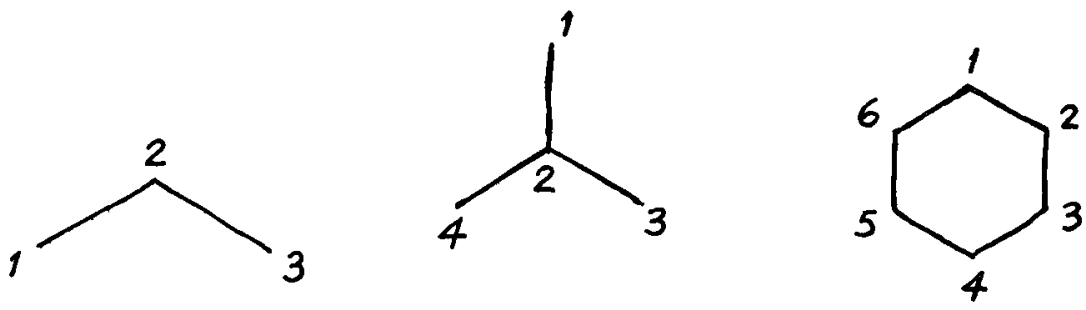

\subsection{Löwdin orthogonalization}

The atomic orbitals are now transformed into LOAO by eq. (1.1), where

$$
\stackrel{T}{\sim} \stackrel{s}{\sim}^{-\frac{1}{2}}=1-\frac{1}{\sim}(s-1)+3 / 8(\underset{\sim}{\sim}-1)^{2}-5 / 16\left(\sim_{\sim}^{s}-1\right)^{3}+\cdots
$$

The series is continued until the transformed overlap matrix is a unit matrix to better than $1 \times 10^{-7}$, that is

$$
\stackrel{S}{\sim}=\underset{\sim}{\sim} \underset{\sim}{\sim}=\stackrel{1}{\sim}
$$

A11 distinct elements of $\underset{\sim}{\sim}$ are listed in Table 2. 


\subsection{Transformed integrals}

The transformed integrals in the LOAO basis, corresponding to $\stackrel{i}{\sim}$ and $\underset{\sim}{\sim}$, are defined by

$$
\begin{aligned}
& I_{i j}=\left\langle\psi_{i},\left(\sum_{\nu \neq j} h_{\nu}\right) \psi_{j}\right\rangle, \\
& F_{i j}=\left\langle\psi_{i}, f \psi_{j}\right\rangle,
\end{aligned}
$$

and

$$
[\mathrm{ab} ; \mathrm{cd}]=\left\langle\psi_{\mathrm{a}}(1) \psi_{\mathrm{c}}(2), g(1,2) \psi_{\mathrm{b}}(1) \psi_{\mathrm{d}}(2)\right\rangle .(2.16)
$$

It follows that the transformed integrals are given simply by

$$
\begin{aligned}
& \underset{\sim}{\sim}=\underset{\sim}{\sim} \underset{\sim}{\sim} \sim \\
& \underset{\sim}{\sim}=\underset{\sim}{\sim} \underset{\sim}{\sim} \underset{\sim}{\sim}=\mathrm{w}_{2 \mathrm{p}} \underset{\sim}{\sim}+\underset{\sim}{\sim},
\end{aligned}
$$

and

$$
[\mathrm{ab} ; \mathrm{cd}]=\sum_{k} \sum_{\ell} \sum_{m} \sum_{n}(\mathrm{k} \ell ; \mathrm{mn}) \mathrm{T}_{k a^{2} \ell \mathrm{b}^{\mathrm{T}} \mathrm{mc}_{\mathrm{nd}}} .
$$

The transformed relative core integrals $I_{m n}$ are listed in Table 3 , and the transformed repulsion integrals [ab;cd] in Tables 4 to 6 . 


\section{Discussion}

The results on repulsion integrals confirm the findings of McWeeny [24] that $[\mathrm{ab} ; \mathrm{cd}]$ is quite small except for the Coulomb type $[\mathrm{aa} ; \mathrm{cc}]$. This justifies the neglect of non-Coulomb-type repulsion integrals in the Pariser-Parr-Pople approach, which is applied with ZDO approximation.

The one-center core integrals show a dependence on the position of the center as well as on the particular molecule. This may cast doubt on the assumption of a single value for the parameter $\alpha$ in the semi-empirical approach. Further work is planned in this direction, as well as in making use of the present results in other calculations, such as starting with LOAO in Coulson-Fischer [25] type wave functions, or in non-paired spatial orbital functions $[10,13]$.

The author is grateful to Professor W. Byers Brown for reading the manuscript. 


\section{References}

[1] Messiah, A。, 1961, Quantum Mechanics, volume 1 (New York: Wiley), p. 173 .

[2] Lowdin, P.-0., 1947, Arkiv. Mat. Astr. Fys., 35A, No. 9.

[3] Car1son, B. C., and Keller, J. M., 1957, Phys. Rev., 105, 102.

[4] Pariser, R., and Parr, R。G., 1953, Jo chem. Phys。, 21, 466, 767.

[5] Pople, J. A., 1953, Trans. Far. Soc. 49, 1375.

[6] Parr, R. G., 1963, The Quantum Theory of Molecular Electronic

Structure (New York: Benjamin).

[7] Lykos, P. G。, 1965, Advances in Quantum Chemistry, volume 1, ed. P. O. Löwdin (New York: Academic)。

[8] Fischer-Hialmas, I., 1965, J. chem. Phys., 42, 1962.

[9] Parr, R. G., and Mulliken, R. S., 1950, J.chem. Phys., 18, 1338.

[10] Hirst, D. M., and Linnett, J. W., 1962, J. chem. Soc., 1035,3844 .

[11] Chong, D. P., and Linnet,

[12] Chong, D P., and Linnett, J. W., 1964, Mol. Phys , 8, 541.

[13] Chong, D. P., and Linnett, J. W., 1965, J. chem. Soc., 1798.

[14] Daude1, R., Lefebure, R., and Moser, C., 1959, Quantum

Chemistry (New York: Interscience), pp. 486ff.

[15] Goeppert-Mayer, M., and Sk1ar, A. L., 1938, J. chem. Phys., $\stackrel{6}{\sim}, 645$.

[16] Sklar, A. L。 and Lyddane, R。H。, 1939, I. chem. Phys., $\stackrel{7}{\sim} 374$. 
References (continued)

[17] Parr, R. G. and Crawford, B. L., 1948, J. chem. Phys., 16, 1049.

[18] Parr, R. G., Craig, D。 P., and Ross, I. Go, 1950, J. chem. Phys. $\stackrel{18}{\sim}, 1561$.

[19] Berthier, G., 1953, J. Chim. phys ., 50, 194.

[20] Lofthus, A., 1961, Mol. Phys., 4, 209.

[21] Guerillot, C. R., Lissillour, R., and Botre1, A., 1965 Theoret. chim. Acta, $2,111$.

[22] Karplus, M., and Shavitt, I。, 1963, J. chem. Phys., 38, 1256.

[23] Roothaan, C. C. J., 1951, J. chem. Phys., 19, 1445.

[24] McWeeny, R., 1954, M. I. T. Solid State and Molecular Group, Quarter 1y Progress Report, $\underset{\sim}{11}, 25$.

[25] Coulson, C. A., and Fischer, I., 1949, Phil. Mag., $\underset{\sim}{\sim}, 386$. 
Table 1 Input integrals

\begin{tabular}{cccc}
\hline $\mathrm{R}_{\mathrm{mn}}$ & $s_{\mathrm{mn}}$ & $\begin{array}{c}(\mathrm{m}: \mathrm{nn}) \\
\text { hartrees }\end{array}$ & $\begin{array}{c}(\mathrm{m}: \mathrm{mn}) \\
\text { hartrees }\end{array}$ \\
\hline $2 \mathrm{~A}$ & 0.2599520 & 0.3010320 & \\
$\sqrt{7 \mathrm{~A}}$ & & 0.0314152 & 0.0693913 \\
$3 \mathrm{~A}$ & & 0.0054776 & \\
$2 \sqrt{3 \mathrm{~A}}$ & 0.03886876 & 0.0019693 & 0.0040005 \\
$\sqrt{13 \mathrm{~A}}$ & & 0.0004879 & \\
$4 \mathrm{~A}$ & 0.01772265 & 0.0003155 & \\
\hline & & 0.0000914 & \\
\hline
\end{tabular}


Table 2, Intermediate results

\begin{tabular}{|c|c|c|c|c|c|}
\hline Matrix & $\mathrm{m}$ & $\mathrm{n}$ & $\begin{array}{l}\text { Allyl } \\
\text { radical }\end{array}$ & $\begin{array}{l}\text { Trimethylene- } \\
\text { methyl radical }\end{array}$ & Benzene \\
\hline \multirow{5}{*}{$\stackrel{i}{\stackrel{i}{\sim}}$} & 2 & 2 & -0.726506 & -1.089760 & \\
\hline & 1 & 1 & -0.572126 & -0.780999 & -1.326948 \\
\hline & 1 & 2 & -0.260763 & -0.330380 & -0.433872 \\
\hline & 1 & 3 & -0.045723 & -0.055801 & -0.075074 \\
\hline & 1 & 4 & & & -0.031044 \\
\hline \multirow{5}{*}{$\stackrel{T}{\sim}$} & 2 & 2 & 1.0543215 & 1.0830548 & \\
\hline & 1 & 1 & 1.0268716 & 1.0270909 & 1.0549169 \\
\hline & 1 & 2 & -0.1377590 & -0.1398510 & -0.1402838 \\
\hline & 1 & 3 & 0.0068517 & 0.0070710 & 0.0094137 \\
\hline & 1 & 4 & & & -0.0063760 \\
\hline
\end{tabular}


Table 3 Transformed relative core integrals, hartrees ${ }^{\dagger}$

\begin{tabular}{lllccc}
\hline Matrix & $\mathrm{m}$ & $\mathrm{n}$ & $\begin{array}{c}\text { Allyl } \\
\text { radical }\end{array}$ & $\begin{array}{r}\text { Trimethylene- } \\
\text { methyl radical }\end{array}$ & Benzene \\
\hline & 2 & 2 & -0.679535 & -1.030421 & \\
$I$ & 1 & 1 & -0.543477 & -0.750688 & -1.275621 \\
& 1 & 2 & -0.100593 & -0.096919 & -0.099020 \\
& 1 & 3 & +0.004214 & +0.003837 & +0.002430 \\
& 1 & 4 & & & -0.002002 \\
\hline
\end{tabular}

+ The sixth decimal place may contain accumulative rounding-off errors. 
Table 4 Transformed repulsion integrals for allyl radica1, hartrees $t$

\begin{tabular}{lllll|lllll}
\hline$a$ & $b$ & $c$ & $d$ & {$[a b ; c d]$} & $a$ & $b$ & $c$ & $d$ & {$[a b ; c d]$} \\
1 & 1 & 1 & 1 & 0.634403 & 1 & 1 & 1 & 3 & -0.000218 \\
2 & 2 & 2 & 2 & 0.647147 & 1 & 1 & 2 & 3 & -0.001863 \\
1 & 1 & 2 & 2 & 0.325417 & 1 & 2 & 1 & 3 & -0.000105 \\
1 & 1 & 3 & 3 & 0.205116 & 1 & 2 & 2 & 3 & -0.000166 \\
1 & 2 & 1 & 2 & 0.003575 & 1 & 3 & 1 & 3 & 0.000051 \\
1 & 1 & 1 & 2 & -0.003415 & 1 & 3 & 2 & 2 & -0.001168 \\
1 & 2 & 2 & 2 & -0.003526 & & & & & \\
\hline
\end{tabular}

+ See footnote, Table 3 
Table 5 Transformed repulsion integrals for trimethylenemethyl radical, hartrees ${ }^{+}$

\begin{tabular}{ccccc|ccccc}
\hline$a$ & $b$ & $c$ & $d$ & {$[a b ; c d]$} & $a$ & $b$ & $c$ & $d$ & {$[a b ; c d]$} \\
\hline 1 & 1 & 1 & 1 & 0.634457 & 1 & 1 & 2 & 3 & -0.001921 \\
2 & 2 & 2 & 2 & 0.661220 & 1 & 1 & 3 & 4 & -0.000854 \\
1 & 1 & 2 & 2 & 0.328231 & 1 & 2 & 1 & 3 & -0.000126 \\
1 & 1 & 3 & 3 & 0.205104 & 1 & 2 & 2 & 3 & -0.000072 \\
1 & 2 & 1 & 2 & 0.003745 & 1 & 2 & 3 & 4 & -0.000096 \\
1 & 1 & 1 & 2 & -0.003526 & 1 & 3 & 1 & 3 & 0.000054 \\
1 & 2 & 2 & 2 & -0.004065 & 1 & 3 & 1 & 4 & 0.000013 \\
1 & 1 & 1 & 3 & -0.000205 & 1 & 3 & 2 & 2 & -0.001086 \\
\hline
\end{tabular}

t See footnote, Table 3 
Table 6 Transformed repulsion integrals for benzene, hartrees ${ }^{\dagger}$

\begin{tabular}{|c|c|c|c|c|c|c|c|c|c|}
\hline a & $\mathrm{b}$ & c & $\mathrm{d}$ & {$[a b ; c d]$} & a & $\mathrm{b}$ & $\mathrm{c}$ & $d$ & {$[\mathrm{ab} ; \mathrm{cd}]$} \\
\hline 1 & 1 & 1 & 1 & 0.647337 & 1 & 1 & 3 & 5 & 0.000744 \\
\hline 1 & 1 & 2 & 2 & 0.328156 & 1 & 2 & 1 & 3 & -0.000130 \\
\hline 1 & 1 & 3 & 3 & 0.205545 & 1 & 2 & 1 & 4 & 0.000058 \\
\hline 1 & 1 & 4 & 4 & 0.179853 & 1 & 2 & 1 & 5 & 0.000091 \\
\hline 1 & 2 & 1 & 2 & 0.003672 & 1 & 2 & 1 & 6 & -0.000176 \\
\hline 1 & 3 & 1 & 3 & 0.000045 & 1 & 3 & 1 & 4 & 0.000001 \\
\hline 1 & 4 & 1 & 4 & 0.000015 & 1 & 3 & 1 & 5 & -0.000001 \\
\hline 1 & 1 & 1 & 2 & -0.003698 & 1 & 2 & 3 & 4 & 0.000244 \\
\hline 1 & 1 & 1 & 3 & 0.000100 & 1 & 2 & 3 & 5 & 0.000010 \\
\hline 1 & 1 & 1 & 4 & -0.000940 & 1 & 2 & 3 & 6 & 0.000057 \\
\hline 1 & 1 & 2 & 3 & -0.002118 & 1 & 2 & 4 & 5 & 0.000092 \\
\hline 1 & 1 & 2 & 4 & 0.000878 & 1 & 3 & 2 & 4 & 0.000019 \\
\hline 1 & 1 & 2 & 5 & 0.000314 & 1 & 3 & 2 & 5 & 0.000008 \\
\hline 1 & 1 & 2 & 6 & -0.001068 & 1 & 3 & 4 & 6 & 0.000005 \\
\hline 1 & 1 & 3 & 4 & 0.000771 & 1 & 4 & 2 & 5 & 0.000003 \\
\hline
\end{tabular}

$\dagger$ See footnote, Table 3 


\author{
Theoretica1 Chemistry Institute \\ University of Wisconsin \\ Madison, Wisconsin
}

Errata, WIS-TCI-102 (1965)

\title{
CORE AND REPULSION INTEGRALS FOR LOWDIN ORTHOGONALIZED \\ ATOMIC ORBITALS IN PI-ELECTRON SYSTEMS ${ }^{+}$
}

by

J. A. Chapman and D. P. Chong

Department of Chemistry, University of British Columbia,

Vancouver 8, B. C., Canada

\footnotetext{
+ This research was supported by the following grants: National Aeronautics and Space Administration Grant NsG-275-62 and National Research Council of Canada Grant A-3582.
} 


\begin{abstract}
A mistake has been discovered in one of the input cards for the case of the trimethylenemethyl radical. This error has led to inaccurate values for most of the integrals for trimethylenemethyl. They should be corrected as follows:

In Table 2 on page 12, the core integral $i_{14}$ should be -0.054641 . The column of entries for trimethylenemethy 1 in Table 3 should read -1.030285, $-0.750656,-0.097256$, and +0.005078. Finally, the entire Table 5 should be replaced by the following one.
\end{abstract}


Table 5 Transformed repulsion integrals for trimethylenemethyl radical, hartrees ${ }^{+}$

\begin{tabular}{cccccccccc}
\hline $\mathrm{a}$ & $\mathrm{b}$ & $\mathrm{c}$ & $\mathrm{d}$ & {$[\mathrm{ab} ; \mathrm{cd}]$} & $\mathrm{a}$ & $\mathrm{b}$ & $\mathrm{c}$ & $\mathrm{d}$ & {$[\mathrm{ab} ; \mathrm{cd}]$} \\
\hline 1 & 1 & 1 & 1 & 0.634454 & 1 & 1 & 2 & 3 & -0.001907 \\
2 & 2 & 2 & 2 & 0.660782 & 1 & 1 & 3 & 4 & -0.000856 \\
1 & 1 & 2 & 2 & 0.328157 & 1 & 2 & 1 & 3 & -0.000113 \\
1 & 1 & 3 & 3 & 0.205102 & 1 & 2 & 2 & 3 & -0.000145 \\
1 & 2 & 1 & 2 & 0.003671 & 1 & 2 & 3 & 4 & -0.000082 \\
1 & 1 & 1 & 2 & -0.003512 & 1 & 3 & 1 & 3 & 0.0000152 \\
1 & 2 & 2 & 2 & -0.003752 & 1 & 3 & 1 & 4 & 0.000011 \\
1 & 1 & 1 & 3 & -0.000207 & 1 & 3 & 2 & 2 & -0.001159 \\
\hline
\end{tabular}

+ See footnote, Table 3 